\title{
Matrix formulæ for Resultants and Discriminants of Bivariate Tensor-product Polynomials
}

\author{
Laurent Busé ${ }^{\mathrm{a}}$ Angelos Mantzaflaris ${ }^{\mathrm{b}}$ Elias Tsigaridas ${ }^{\mathrm{c}}$ \\ ${ }^{a}$ Université Côte d'Azur, Inria, France \\ ${ }^{\mathrm{b}}$ Radon Institute for Computational and Applied Mathematics (RICAM), Austrian \\ Academy of Sciences, Altenberger Str. 69, 4040, Linz, Austria \\ 'Sorbonne Universités, UPMC Univ Paris 06, CNRS, INRIA, Laboratoire \\ d'Informatique de Paris 6 (LIP6), Équipe PolSys, 4 place Jussieu, 75252 Paris \\ Cedex 05, France
}

\begin{abstract}
The construction of optimal resultant formulæ for polynomial systems is one of the main areas of research in computational algebraic geometry. However, most of the constructions are restricted to formulæ for unmixed polynomial systems, that is, systems of polynomials which all have the same support. Such a condition is restrictive, since mixed systems of equations arise frequently in many problems. Nevertheless, resultant formulæ for mixed polynomial systems is a very challenging problem.

We present a square, Koszul-type, matrix, the determinant of which is the resultant of an arbitrary (mixed) bivariate tensor-product polynomial system. The formula generalizes the classical Sylvester matrix of two univariate polynomials, since it expresses a map of degree one, that is, the elements of the corresponding matrix are up to sign the coefficients of the input polynomials. Interestingly, the matrix expresses a primal-dual multiplication map, that is, the tensor product of a univariate multiplication map with a map expressing derivation in a dual space. In addition we prove an impossibility result which states that for tensor-product systems with more than two (affine) variables there are no universal degree-one formulæ, unless the system is unmixed. Last but not least, we present applications of the new construction in the efficient computation of discriminants and mixed discriminants.
\end{abstract}

Key words: Koszul resultant matrix, tensor-product, singular locus, eliminant, mixed discriminant, mixed polynomial system, resultant

Email addresses: laurent.buse@inria.fr (Laurent Busé), 


\section{Introduction}

One of the central problems in elimination theory is the construction of determinantal formulæ for the resultant. In this context there is also a special emphasis on exploiting the sparsity of the input, or in other words the support, of the involved polynomials.

We refer the interested reader to [25] as a basic reference, to [5] for a nice introduction to the theory of resultants, to [3] for its application in Computeraided Geometric Design (CAGD), and to [21] for various matrix constructions. Among the various constructions the best we can hope for is a degree-one formula; that is a matrix whose non-zero entries are coefficients of the input polynomials (modulo multiplication with \pm 1 ), and whose determinant is equal to the resultant. The Sylvester-type formulæ fall in this category. Unfortunately, such formulæ do not always exist for given Newton polytopes. There are also Bézout-type formulæ where the entries of the matrix are coefficients of the Bézoutian polynomial and thus they are high degree polynomials in the coefficients of the input polynomials. We call the matrices that have entries that are both Sylvester-type and Bézoutian-type, hybrid. For general algorithms for computing resultant matrices, let us mention [11] that proposes a rational resultant formula (quotient of two determinants) for the mixed homogeneous case, and [10] that generalizes Macaulay's construction [29] to the sparse case, as well as the computational tools in [4, 22, 23].

We focus on resultants and discriminants for polynomial systems in two variables. A polynomial system is unmixed if all of its polynomials have the same Newton polytope, and mixed otherwise. Exact resultant formulæ are mostly known for certain classes of unmixed systems, and very little is known for the general mixed case. We are interested in optimal (or exact) degree-one formulæ for the mixed resultant. Degree-one formulæ refer to matrices where the entries are coefficients of the input polynomials (possibly with a sign change). This kind of expressions are very convenient for both the analysis and the implementation of resultant methods, since the matrix entries are simple to compute and have bitsize that matches the bitsize of the input. Common degree-one formulæ are the Sylvester-type formulæ; in this work we present a different one that expresses a second order Koszul map.

Khetan [27] presented explicit exact formulæ for an arbitrary unmixed sparse bivariate polynomial system. His determinantal formula is a hybrid Sylvester and Bézout type. Also in the unmixed case there are necessary and sufficient conditions for the Dixon resultant formulation to produce the resultant $[7,8]$. In the same context, Elkadi and Galligo proposed in [17] to use a variable

angelos.mantzaflaris@oeaw.ac.at (Angelos Mantzaflaris), elias.tsigaridas@inria.fr (Elias Tsigaridas). 
substitution and two iterated resultants to compute the resultant polynomial.

Regarding Sylvester-type formulæ, in [43] matrices expressing optimally the resultant of unmixed bivariate polynomials with corner-cut support are found. Moreover, Sylvester formulæ for more general unmixed bivariate systems were depicted in [28]. The proof of the main theorem makes use of tools from algebraic geometry, including sheaf cohomology on toric varieties and Weyman's resultant complex.

There are also methods for constructing resultant matrices for bivariate polynomial systems that combine Sylvester type blocks with toric Jacobian blocks in the case where the Newton polytopes of the polynomials are scaled copies of a single polygon, see [12] and references therein. The determinant of these matrices is a multiple of the sparse resultant, that is, the formula might not be optimal.

Resultants are closely related to discriminants. Discriminants have many applications, ranging from singularity theory of partial differential equations to the computation of the Voronoï diagram of curved objects [6, 19, 24]. Especially for the bivariate case we refer to [15] and references therein, that relates the mixed discriminant with the sparse resultant and the toric Jacobian.

Tensor-product systems fall in the general category of multihomogenenous systems [39]. In [36] and [33] one finds the first expressions of the resultant of such systems as the determinant of a matrix.

For unmixed multigraded systems Sturmfels and Zelevinski provided in [38] optimal Sylvester-type formulæ. These formulæ arise as certain choices of a Weyman complex of modules [25, 40, 41]. Many, if not all, classical resultant matrices are instances of such complexes [42], including the projective resultant $[11]$. In $[14,20]$ there is a systematic exploration of possible determinantal complexes, and also a software package that produces formulæ for unmixed (and even scaled) resultants. Interestingly, there is a plethora of hybrid resultant matrices that consist of Bézout-type and Sylvester-type blocks [14, 20], see also [9].

The main contributions of this work are as follows. We present a determinantal degree-one formula for the resultant of arbitrary (mixed) bivariate tensorproduct systems (Theorem 3.2). The formula applies without any restrictions on the bidegree of the polynomials, it expresses a Koszul map and has degree one with respect to the coefficients of the system. Moreover, we prove that the univariate and the bivariate case are the only cases among tensor-product polynomials which admit an unconditional formula of degree one (Lemma 3.3). We provide a constructive method to compute the new matrix, by identifying the (dual) multiplication maps, therefore making our formula explicit (Theorem 4.1). We call the matrix Koszul resultant matrix. As a consequence of our 
matrix, we provide new formulæ for computing the discriminant of one or two bivariate tensor-product polynomials. Compared to the existing literature, we reduce significantly the degree of the extraneous factor which is involved in the computation. Another important aspect is that our formulæ provided are free of nonzero multiplicative constant, so that they yield smoothness criteria that are valid in arbitrary characteristic.

The rest of the paper is organized are follows. In the next section we present preliminary results that we need for our construction. In Section 3 we present the mixed resultant complex for the bivariate case and we derive the determinantal Koszul formula. Moreover, we show that universal degree-one formulæ arise for at most two variables. In Section 4 we provide the algorithmic construction of the Koszul resultant matrix, by identifying the cohomology groups which appear in the complex. Finally, in Section 5 we apply the new matrix to the problem of computing discriminants and mixed discriminants. Throughout the paper, several toy examples accompany the main results.

A preliminary version of this paper appeared in [32]. In the current final version the Sections 4 and 5 are expanded significantly, and in particular the computation of (mixed) discriminants is improved by relating them with mixed resultants of lower degree.

\section{Preliminaries}

Let $S_{1}\left(b_{1}\right)$, resp. $S_{2}\left(b_{2}\right)$, be the linear space of univariate polynomials of degree $b_{1}$, resp. $b_{2}$, in $\mathbb{K}[x]$, resp. $\mathbb{K}[y]$, where $\mathbb{K}$ is an algebraically closed field. The tensor-product space $S_{1}\left(b_{1}\right) \otimes S_{2}\left(b_{2}\right) \subset \mathbb{K}[x, y]$ consists of all polynomials of bidegree $\left(b_{1}, b_{2}\right)$. Throughout the paper we consider three arbitrary bivariate tensor-product polynomials

$$
f_{0}, f_{1}, f_{2} \in \mathbb{K}[x, y], \text { with } f_{i} \in S_{1}\left(d_{i 1}\right) \otimes S_{2}\left(d_{i 2}\right)
$$

and we denote by $\boldsymbol{d}_{i}=\left(d_{i 1}, d_{i 2}\right)$ the bidegree of $f_{i}, \in\{0,1,2\}$. In matrix notation, we can write each polynomial as

$$
f_{i}(x, y)=\left(1, x, x^{2}, \ldots, x^{d_{i 1}}\right) C_{i}\left(1, y, y^{2}, \ldots, y^{d_{i 2}}\right)^{T},
$$

where $C_{i} \in \mathbb{Z}^{\left(d_{i 1}+1\right) \times\left(d_{i 2}+1\right)}$ are the coefficient matrices, $i \in\{0,1,2\}$.

If we assume that the square system $\left\{f_{i}=f_{j}=0\right\}$ is zero-dimensional, then the number of its roots in $\mathbb{P}^{1} \times \mathbb{P}^{1}$ equals the Bézout bound, that is

$$
D=D\left(f_{i}, f_{j}\right):=d_{i 1} d_{j 2}+d_{j 1} d_{i 2}
$$




$$
\begin{aligned}
& \nu: \quad 3 \quad 2 \quad 1 \quad 00-1 \quad-2 \\
& 0 \mathcal{H}^{0}\left(\boldsymbol{m}-\boldsymbol{\ell}_{012}\right) \rightarrow \bigoplus_{i j} \mathcal{H}^{0}\left(\boldsymbol{m}-\boldsymbol{\ell}_{i j}\right) \rightarrow \bigoplus_{i} \mathcal{H}^{0}\left(\boldsymbol{m}-\boldsymbol{d}_{i}\right) \rightarrow \quad, \quad \rightarrow \mathcal{H}^{0}(\boldsymbol{m}) \\
& \oplus \quad \nearrow \quad \oplus, i^{\prime} \oplus \\
& 1
\end{aligned}
$$

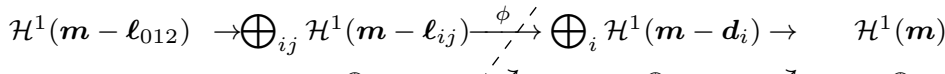

$$
\begin{aligned}
& 2 \\
& \mathcal{H}^{2}\left(\boldsymbol{m}-\boldsymbol{\ell}_{012}\right) \quad \rightarrow \bigoplus_{i j} \mathcal{H}^{2}\left(\boldsymbol{m}-\boldsymbol{\ell}_{i j}\right) \rightarrow \bigoplus_{i} \mathcal{H}^{2}\left(\boldsymbol{m}-\boldsymbol{d}_{i}\right) \rightarrow \mathcal{H}^{2}(\boldsymbol{m})
\end{aligned}
$$

Fig. 1. The mixed Weyman complex of bivariate tensor-product polynomials. Whenever $i$ or $i j$ appear in a sum, they take values over all subsets of $\{0,1,2\}$ of 1 and 2 elements, respectively. The term $K_{\nu, q}$ in (4) is shown in row $q$ and column $\nu$ in this diagram. The resultant formula of Theorem 3.2 is the map $\phi: K_{1,1} \rightarrow K_{0,1}$, and is also marked in the diagram. For the sake of brevity we define $\boldsymbol{\ell}_{012}=\boldsymbol{d}_{0}+\boldsymbol{d}_{1}+\boldsymbol{d}_{2}$ and $\boldsymbol{\ell}_{i j}=\boldsymbol{d}_{i}+\boldsymbol{d}_{j}$. Determinantal formulæ arise whenever $K_{2, q}=K_{-1, q}=0$, for all $q \in\{0,1,2\}$. The map $K_{\nu, q} \rightarrow K_{\nu-1, q^{\prime}}$ has degree one if and only if $q=q^{\prime}$, and is always zero if $q<q^{\prime}$, this is why no arrows point downwards in the diagram. The arrow between $K_{1,2}$ and $K_{0,0}$ is drawn with a dashed line for the sake of clarity.

where $D$ is the coefficient of the multilinear term of a certain polynomial [34, Theorem 2]. This number is the mixed volume of the system and, in the case of infinitely many roots, equals the degree of the variety [18].

Example 2.1. Consider two tensor-product polynomials of degrees $\boldsymbol{d}_{1}=(1,2)$ and $\boldsymbol{d}_{2}=(2,1)$ and

$$
\begin{aligned}
& f_{1}=b_{1,2} x y^{2}+b_{0,2} y^{2}+b_{1,1} x y_{1}+b_{0,1} y+b_{1,0} x+b_{0,0} \\
& f_{2}=c_{2,1} x^{2} y+c_{1,1} x y+c_{2,0} x^{2}+c_{0,1} y_{1}+c_{1,0} x+c_{0,0}
\end{aligned}
$$

The number of roots of the system in $\mathbb{P}^{1} \times \mathbb{P}^{1}$ is $D\left(f_{1}, f_{2}\right)=5$.

We fix the bidegrees in Eq. (1) and we consider the vector space

$$
\mathcal{V}:=\bigoplus_{i=0}^{2} S_{1}\left(d_{i 1}\right) \otimes S_{2}\left(d_{i 2}\right) \cong \prod_{i=0}^{2} \mathbb{P}^{d_{i 1}} \times \mathbb{P}^{d_{i 2}}
$$

where we assume it yields a very ample vector bundle of rank 3 on the irreducible projective variety $X=\mathbb{P}^{1} \times \mathbb{P}^{1}[25]$. This means that $d_{i j} \geq 1$ for all $i, j$. We consider the incidence variety

$$
\mathcal{W}=\left\{\left(C_{0}, C_{1}, C_{2}, x, y\right) \in \mathcal{V} \times \mathbb{P}^{1} \times \mathbb{P}^{1}: f_{i}(x, y)=0, i=0,1,2\right\}
$$

and the projection $\pi: \mathcal{W} \rightarrow \mathcal{V}$. The image

$$
\pi(\mathcal{W})=\left\{\left(C_{0}, C_{1}, C_{2}\right): \exists(x, y), f_{i}(x, y)=0\right\}
$$

is the resultant variety and is an irreducible hypersurface in $\mathcal{V}$. The equation $\mathcal{R}\left(f_{0}, f_{1}, f_{2}\right)=0$ of this hypersurface characterizes systems as in Eq. (1) with 
common roots in $X$. This polynomial in $\mathbb{Z}[\mathcal{V}]$ is the resultant of $f_{0}, f_{1}, f_{2}$, it is homogeneous of degree $D\left(f_{i}, f_{j}\right)$ with respect to (the coefficients of) $f_{k}$, $\{i, j, k\}=\{0,1,2\}$, and therefore it has total degree

$$
\operatorname{deg} \mathcal{R}_{\boldsymbol{d}_{0}, \boldsymbol{d}_{1}, \boldsymbol{d}_{2}}\left(f_{0}, f_{1}, f_{2}\right)=\sum_{0 \leq i<j \leq 2} d_{i 1} d_{j 2}+d_{j 1} d_{i 2} .
$$

This resultant is an instance of the sparse resultant [25], where the toric variety is the product of two projective lines. Our aim is to obtain a square matrix, the entries of which are coefficients, up to a sign, of the coefficients of the input polynomials, that has determinant equal to the resultant.

\section{Mixed determinantal complex}

In this section we elaborate on a mixed determinantal Weyman complex, which will help us to construct a Koszul resultant matrix. We can compute the resultant polynomial as the determinant of the Weyman complex [40], see also [2], which arises by applying the, so called, geometric technique of [41] to the incidence variety of $f_{0}, f_{1}, f_{2}$ and generalizes the Cayley-Koszul complex [25]. Note that we cannot use the classical Koszul complex to derive resultant matrices for tensor-product (or, more generally, bigraded) polynomial systems, cf. [37].

\subsection{The parameterized complex}

For any $\boldsymbol{m}=\left(m_{1}, m_{2}\right) \in \mathbb{Z}^{2}$ and $\left(f_{0}, f_{1}, f_{2}\right) \in \mathcal{V}$, Weyman's construction is a complex $K_{\bullet}=K_{\bullet}\left(m_{1}, m_{2}\right)$ of finite dimensional vector spaces:

$$
0 \rightarrow K_{3} \rightarrow K_{2} \rightarrow K_{1} \stackrel{\phi}{\rightarrow} K_{0} \rightarrow K_{-1} \rightarrow K_{-2} \rightarrow 0 \text {. }
$$

The terms are defined as $K_{\nu}=\oplus_{q=0}^{2} K_{\nu, q}$ with

$$
K_{\nu, q}=\bigoplus_{|I|=\nu+q} \mathcal{H}^{q}\left(\boldsymbol{m}-\sum_{i \in I} \boldsymbol{d}_{i}\right) \quad, \quad \nu=-2, \ldots, 3
$$

where $I$ runs over all subsets of $\{0,1,2\}$ of cardinality $\nu+q$. We present the complex in Figure 1 (the indices $\nu, q$ refer to columns and rows, respectively). We use the notation $\boldsymbol{\ell}_{i j}$ and $\boldsymbol{\ell}_{012}$ to denote sums of degrees of the $f_{i}$ 's.

Since we are working in the product of two projective lines, $X=\mathbb{P}^{1} \times \mathbb{P}^{1}$, the cohomology groups take the form

$$
\mathcal{H}^{q}(\boldsymbol{b})=H^{a_{1}}\left(b_{1}\right) \otimes H^{a_{2}}\left(b_{2}\right),
$$


with $a_{1}+a_{2}=q, \nu=-2, \ldots, 3$, and $H^{a}(b), b \in \mathbb{Z}$ denotes the $a$-th cohomology group of $\mathbb{P}^{1}$ with coefficients in the sheaf $\mathscr{O}(b)$ [26].

These terms depend solely on $m_{1}, m_{2}$. The maps between the terms (e.g. $\phi)$ depend polynomially on the coefficients of $f_{0}, f_{1}, f_{2}$. This construction appeared in [40] and a detailed presentation is given in [41].

The complex appears in Figure 1 in its full generality. However, several of the terms $K_{\nu, q}$ can be zero, but not all of them. We observe that for any integer vector $\boldsymbol{m}$, it holds $K_{1} \neq 0$ and $K_{0} \neq 0$. Indeed, due to Bott's formula [1] the dimension of a cohomology group as a $\mathbb{K}$-vector space is

$$
\operatorname{dim} H^{a}(b)=\left((-1)^{a}(b+1)\right)_{+} \quad, \quad a \in\{0,1\},
$$

where for an integer $q \in \mathbb{Z},(q)_{+}:=(q+|q|) / 2$.

Whenever this dimension is zero, it is understood that $H^{a}(b)=0$. This is the case for negative integers for global sections $(a=0)$, and for integers $b \geq-1$ of the first order cohomologies.

The crucial property of the complex (3) is that its determinant is equal, up to sign, to $\mathcal{R}_{\boldsymbol{d}_{0}, \boldsymbol{d}_{1}, \boldsymbol{d}_{2}}\left(f_{0}, \ldots, f_{2}\right)$. This is proved in $[25,42]$ under the very ampleness assumption, i.e. $d_{i j} \geq 1$ for all $i, j$. In general, this is a rational expression of determinants of maximal minors of differentials [25]. When the complex has only two non-zero terms (for specific integers $m_{1}, m_{2}$ ), then we obtain the resultant as the determinant of a single square matrix expressing the map $\phi$ at the non-zero part of the complex. We call such complexes and the induced square matrix expressions determinantal. In this case the determinantal complexes are of the form

$$
0 \rightarrow K_{1} \rightarrow K_{0} \rightarrow 0
$$

that is $\nu \in\{0,1\}$.

The linear map $\phi$ is an epimorphism if and only if the complex is exact or, equivalently, the polynomials do not have a common root in $\mathbb{P}^{1} \times \mathbb{P}^{1}$. The possible values of $\left(m_{1}, m_{2}\right)$ which lead to determinantal complexes is a finite set $[14,20]$.

Example 3.1. The resultant of three bilinear forms, that is $\boldsymbol{d}_{0}=\boldsymbol{d}_{1}=\boldsymbol{d}_{2}=$ $(1,1)$, corresponds to the determinantal complex $K_{\bullet}(2,1)$, where $\boldsymbol{m}=(2,1)$. Using (12), the complex (3) becomes $K_{1,1} \stackrel{\phi}{\rightarrow} K_{0,0}$, and implies the existence of a map $\phi: S(1,0)^{\oplus 3} \rightarrow S(2,1)$ expressing the resultant. This resultant matrix is depicted in [14, Section 7.1].

Many classically known resultant formulæ can be obtained as the determinant of different instances of $\phi$ in (3), for appropriate choices of integers 
$\left(m_{1}, m_{2}\right) \in \mathbb{Z}^{2}$. Moreover, the existence of a determinantal complex implies a determinantal formula for the resultant.

A Sylvester map is a linear function $S: \mathcal{P} \rightarrow \mathcal{Q}$ and has the form

$$
\left(g_{0}, g_{1}, g_{2}\right) \mapsto g_{0} f_{0}+g_{1} f_{1}+g_{2} f_{2} .
$$

When $\operatorname{dim} \mathcal{P}=\operatorname{dim} \mathcal{Q}$ this map is a generically surjective linear operator which can be expressed as the matrix (in compact form)

$$
\left[\begin{array}{lll}
f_{0} & f_{1} & f_{2}
\end{array}\right]
$$

The determinant of this matrix is equal to $\mathcal{R}\left(f_{0}, f_{1}, f_{2}\right)$.

A Koszul map (see for instance [35]) $K: \mathcal{P} \rightarrow \mathcal{Q}$ of second order is a map of the form

$$
\left(g_{0}, g_{1}, g_{2}\right) \mapsto\left(-g_{0} f_{1}-g_{1} f_{2}, g_{0} f_{0}-g_{2} f_{2}, g_{1} f_{0}+g_{2} f_{1}\right)
$$

Similarly, in the case of a linear operator we shall recover the resultant of $f_{0}, f_{1}, f_{2}$ as the determinant of the matrix

$$
\left[\begin{array}{rrr}
-f_{1} & -f_{2} & 0 \\
f_{0} & 0 & -f_{2} \\
0 & f_{0} & f_{1}
\end{array}\right] \text {. }
$$

In the next section we present the construction of a general Koszul operator for bivariate tensor-product systems.

\subsection{The determinantal Koszul formula}

In this section we identify a determinantal complex with a linear differential $\phi$ which has degree one with respect to the coefficients of the input polynomials, and whose determinant is the resultant of the system. The matrix expresses a Koszul linear operator, thus the name Koszul formula and Koszul-type matrices.

We can obtain a non-hybrid determinantal formula if and only if the non-zero terms in (3) (see also Figure 1) are

$$
0 \rightarrow K_{1, q} \stackrel{\phi}{\rightarrow} K_{0, q^{\prime}} \rightarrow 0
$$

for some $q, q^{\prime} \in\{0,1,2\}$ (cf. [42]). General such formulæ for unmixed multilinear systems are identified in $[14,20,38,42]$. In general, the degree of 
$\phi: K_{\nu, q} \rightarrow K_{\nu-1, q^{\prime}}$ with respect to $f_{0}, f_{1}, f_{2}$ is equal to $\left(q-q^{\prime}+1\right)_{+}$therefore degree one linear maps arise from $q=q^{\prime}$. The following theorem presents the degree vector and formula of a degree one $\left(q=q^{\prime}=1\right)$ determinantal complex for the systems in question.

Theorem 3.2. Consider the degree vector

$$
\boldsymbol{m}=\left(d_{01}+d_{11}+d_{21}-1,-1\right) .
$$

The non-zero part of the complex $K_{\bullet}(\boldsymbol{m})$ is the operator $\phi: K_{1,1} \rightarrow K_{0,1}$, that is,

$$
\phi: \bigoplus_{0 \leq i<j \leq 2} \mathcal{H}^{1}\left(\boldsymbol{m}-\boldsymbol{\ell}_{i j}\right) \rightarrow \bigoplus_{0 \leq i \leq 2} \mathcal{H}^{1}\left(\boldsymbol{m}-\boldsymbol{d}_{i}\right) .
$$

Proof. First we show that $\mathcal{H}^{2}(\boldsymbol{m})=\mathcal{H}^{0}(\boldsymbol{m})=0$ (cf. Figure 1). Indeed we note that, by $(5), H^{0}(b)$ vanishes for any $b \leq-1$, therefore the row $q=0$ vanishes if $m_{k} \leq-1$, for some $k$. Similarly, $H^{1}(b)$ vanishes for any $b \geq-1$, so the row $q=2$ vanishes if there exists $k$ s.t. $m_{k} \geq d_{0 k}+d_{1 k}+d_{2 k}-1$.

Therefore the complex is confined to the row $q=1$. We can repeat these arguments to see that the extremal terms of the row $q=1$ also vanish, that is, $\mathcal{H}^{1}\left(\boldsymbol{m}-\boldsymbol{\ell}_{012}\right)=\mathcal{H}^{1}\left(-1,-d_{02}-d_{12}-d_{22}-1\right)=0$ and $\mathcal{H}^{1}(\boldsymbol{m})=0$. We are left with the map (9), which is actually an operator, as we can verify using Bott's formula (5).

We remark that another choice of degree vector is possible, that is, $\boldsymbol{m}=$ $\left(-1, d_{02}+d_{12}+d_{22}-1\right)$, which leads to the transposed operator of (9) (essentially the same formula).

In the univariate case the resultant variety is determinantal; it is given by the classical Sylvester matrix of the form (6). We have just shown that, in the bivariate tensor-product case, there exists a formula, which does not have the structure of (6). A natural question is whether any of these two (or any other) degree-one formulæ are possible, unconditionally, for multivariate tensor-product systems.

Lemma 3.3. For $n>2$, and for tensor-product polynomials $f_{0}, \ldots, f_{n}$ with $n$ variables there is no universal degree one determinantal formula for their resultant. That is, the only such formulæ, without any assumption on the degrees $\boldsymbol{d}_{i}$ are the classical Sylvester map for $n=1$ and the Koszul map (9) for $n=2$.

Proof. For proving that the only universal degree one formulæ formulæ arise for $n \leq 2$, it suffices to depict a general family of polynomials for which no 
such formula is possible. To this end, consider a degree vector $\boldsymbol{v} \in \mathbb{Z}_{\geq 0}$ and let the degrees be $\boldsymbol{d}_{i}=s_{i} \boldsymbol{v}, 0 \leq i \leq n$, for some positive integers $s_{0}, s_{1}, \ldots, s_{n}$ (scaled Newton polytopes). Indeed, in [20, Theorem 4.11] it is proven that the only degree one ${ }^{1}$ formulæ (up to transposition) for this class of systems arise for $n \leq 2$. These are the classical Sylvester map (for $n=1$ ) and the Koszul map of (9) (for $n=2$ ).

Nevertheless the determinantal Koszul formula does appear in certain cases for $n>2$. For example, for trivariate tensor-product polynomials of degrees $\boldsymbol{d}_{0}=\boldsymbol{d}_{1}=\boldsymbol{d}_{2}=(1,1,2)$ and $\boldsymbol{d}_{3}=(1,1,4)$ the formula (9) is determinantal, for $\boldsymbol{m}=(2,3,-1)$. However, if we change $\boldsymbol{d}_{3}$ to $(2,2,4)$ the system is scaled, and for instance the degree vector $\boldsymbol{m}=(2,4,-1)$ yields the determinantal complex

$$
\begin{aligned}
0 & \rightarrow \mathcal{H}^{1}(0,2,-5)^{\oplus 3} \oplus \mathcal{H}^{2}(-2,0,-9)^{\oplus 3} \\
& \rightarrow \mathcal{H}^{1}(1,3,-3)^{\oplus 3} \otimes \mathcal{H}^{1}(0,2,-5) \rightarrow 0
\end{aligned}
$$

which does contain a Koszul part, but also additional degree two differentials appear.

Finally, for the sake of completeness we briefly recall the (mixed) homogeneous case. Consider homogeneous bivariate polynomials of degrees $d_{0}, d_{1}, d_{2}$, in non-decreasing order. In [11, Lemma 5.3] it is shown that a determinantal formula is possible if and only if

$$
1+d_{0}+d_{1}-d_{2} \geq 0
$$

Moreover, [20, Theorem 4.11] applies, since this system is trivially in the class of systems with scaled Newton polytopes, and it implies that arbitrary mixed homogeneous systems do not admit any degree-one formulæ, except if $n=1$.

If all the polynomials have the same bidegree then another set of $n$ ! Sylvester formulæ exist, for any $n$. These were discovered in [38] and independently in [28] for the bivariate case. Let $\boldsymbol{d}_{0}=\boldsymbol{d}_{1}=\boldsymbol{d}_{2}=\left(a_{1}, a_{2}\right)$, so that $D\left(f_{i}, f_{j}\right)=$ $2 a_{1} a_{2}$; the Newton polytopes $\left(a_{1}, 2 a_{2}\right)$ and $\left(2 a_{1}, a_{2}\right)$ both have volume $2 a_{1} a_{2}$ and multiplication by $f_{i}$ results in polytopes $\left(2 a_{1}, 3 a_{2}\right)$ and $\left(3 a_{1}, 2 a_{2}\right)$ (respectively) with volume $6 a_{1} a_{2}$. In our terminology, the complex $K_{\bullet}\left(2 a_{1}, 3 a_{2}\right)$ is determinantal and of Sylvester-type. Lemma 3.3 implies that these degree vectors are not universal, that is, the assumptions of [28, Theorem 1] cannot be satisfied by arbitrary degree vectors $\boldsymbol{d}_{i}$. Moreover, our Koszul formula does not satisfy these assumptions; however, it provides an additional formula for the resultant of unmixed systems.

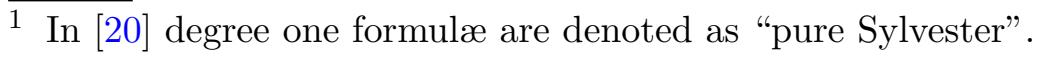


Example 3.4. Consider three polynomials with common bidegree $\boldsymbol{d}_{0}=\boldsymbol{d}_{1}=$ $\boldsymbol{d}_{2}=(2,3)$. There exist two determinantal Sylvester formulæ, for $\boldsymbol{m}=(5,5)$ and $\boldsymbol{m}=(3,8)$ as well as the Koszul determinantal formula $\boldsymbol{m}=(5,-1)$. These three formulæ all have (matrix) dimension $24 \times 24$, which is the same as the degree of the resultant as a polynomial in $\mathbb{Z}[\mathcal{V}]$.

\section{The assembly of the Koszul-type matrix}

In this section we provide the algorithmic construction of the Koszul resultant matrix.

\subsection{Primal-dual multiplication maps}

First, we define dual polynomial spaces. Let $S_{i}^{*}(d)$ be the dual space of $S_{i}(d)$, that is, the space of linear functionals

$$
\lambda: S_{i}(d) \rightarrow \mathbb{K}
$$

We focus on the $y$ variable $(i=2)$ for the rest of the presentation. This space is isomorphic to (evaluations of) polynomials in formal derivatives, formally $S_{2}^{*}(d) \cong \mathbb{R}\left[\partial_{y}\right]_{d}$, see $[30,31]$ and references therein for a detailed presentation. We use this identification to obtain a basis for $S_{2}^{*}(d)$. For a univariate polynomial $g \in \mathbb{K}[y]$ and for $j \in \mathbb{Z}_{\geq 0}$ we define the functionals (reciprocal monomials)

$$
\psi^{-j} \in \mathbb{R}\left[\partial_{y}\right] \quad \text { with } \quad \psi^{-j}(g):=\frac{1}{j !} \frac{\mathrm{d}^{j} g}{\mathrm{~d} y^{j}}(0)
$$

where in particular $\psi^{0}(g):=1_{*}(g)=g(0)$ is evaluation at 0 . This way we obtain a basis $S_{2}^{*}(d)=\left\langle 1_{*}, \psi^{-1}, \ldots, \psi^{1-d}\right\rangle$. The latter is a $\mathbb{K}[y]$-module, equipped with the operation

$$
y^{b} \cdot \psi^{-j}:= \begin{cases}\psi^{(b-j)} & , \text { if } b \leq j \\ 0 & , \text { otherwise }\end{cases}
$$

Here $\psi^{-j}$ acts as the inverse of $y^{j}$ in $\mathbb{K}\left[\partial_{y}\right]$, and this representation of $S_{2}^{*}(d)$ is sometimes referred to as inverse system.

The building blocks of the Koszul operator are (tensor products of): 
- multiplication maps

$$
M_{h_{1}}: S_{1}\left(a_{1}\right) \rightarrow S_{1}\left(a_{1}+b_{1}\right)
$$

with $g \mapsto h_{1} g$ expressing the multiplication by a polynomial $h_{1} \in S_{1}\left(b_{1}\right)$,

- adjoint multiplication maps

$$
M_{h_{2}}^{*}: S_{2}^{*}\left(a_{2}+b_{2}\right) \rightarrow S_{2}^{*}\left(a_{2}\right)
$$

with $\lambda \mapsto h_{2} \cdot \lambda$, expressing the action of a polynomial $h_{2} \in S_{2}\left(b_{2}\right)$, as defined in (10).

We now recall the tensor product of multiplication and adjoint multiplication. With the preceeding definitions, there exists a unique linear operator

$$
\delta_{h_{1}, h_{2}}: S_{1}\left(a_{1}\right) \otimes S_{2}^{*}\left(a_{2}+b_{2}\right) \rightarrow S_{1}\left(a_{1}+b_{1}\right) \otimes S_{2}^{*}\left(a_{2}\right)
$$

satisfying

$$
\delta_{h_{1}, h_{2}}\left(x^{i} \otimes \psi^{-j}\right)=M_{h_{1}}\left(x^{i}\right) \otimes M_{h_{2}}^{*}\left(\psi^{-j}\right) .
$$

This map inherits the properties of its components, most importantly injectivity or surjectivity. In what follows we will omit the " $\otimes$ " and "." between monomials and reciprocal monomials for the sake of brevity. With this definition of the tensor-product operator, we obtain

$$
\left(x^{a} y^{b}\right)\left(x^{i} \psi^{-j}\right)= \begin{cases}x^{a+i} \psi^{(b-j)} & , \text { if } b \leq j \\ 0 & , \text { otherwise }\end{cases}
$$

The above action extends by linearity to any element of $S_{1}\left(a_{1}\right) \otimes S_{2}^{*}\left(a_{2}+b_{2}\right)$. Moreover, map $\delta_{x^{a}, x^{b}}$ expresses the multiplication by the monomial $x^{a} y^{b}$ in that tensor-product space; by linearity we can consider any $h \in S_{1}\left(b_{1}\right) \otimes S_{2}\left(b_{2}\right)$ and define $\delta_{h}$ using (11). We refer to maps of the form of $\delta_{h}$ as primal-dual multiplication maps, see also [25, Ch. 13].

\subsection{The Koszul resultant matrix}

We are now in position to construct the final matrix. The cohomology groups in (4) are identified as

$$
H^{a}(b) \cong \begin{cases}S_{i}(b) & , a=0 \text { and } b>-1 \\ S_{i}^{*}(-b-2) & , a=1 \text { and } b<-1 \\ 0 & , a \neq 0,1 \text { or } b=-1\end{cases}
$$


This identification will allow us to deduce monomial bases for $H^{a}(b)$ and to express the map $\phi$ between the modules of (9) as a matrix. Indeed, we define

$$
V_{i j}:=\mathcal{H}^{1}\left(\boldsymbol{m}-\boldsymbol{\ell}_{i j}\right) \cong S_{1}\left(d_{k 1}-1\right) \otimes S_{2}^{*}\left(d_{i 2}+d_{j 2}-1\right)
$$

with $i<j$ and $k$ such that $\{i, j, k\}=\{0,1,2\}$ and

$$
W_{k}:=\mathcal{H}^{1}\left(\boldsymbol{m}-\boldsymbol{d}_{k}\right) \cong S_{1}\left(d_{i 1}+d_{j 1}-1\right) \otimes S_{2}^{*}\left(d_{k 2}-1\right),
$$

where $\boldsymbol{m}$ as in (8). Consider the primal-dual multiplication maps (see paragraph 4.1)

$$
\delta_{i j}^{r}: V_{i j} \rightarrow W_{r}, \text { for all } i, j \in\{0,1,2\}, i<j \text { and } r \in\{i, j\},
$$

expressing multiplication by $f_{s}$ where $\{s\}=\{i, j\} \backslash\{r\}$. We will use the same notation for $\delta_{i j}^{r}$ and its (monomial basis) matrix representation. We have the following

Theorem 4.1. Let $f_{0}, f_{1}, f_{2}$ be three bivariate tensor-product polynomials of as in (1) such that $d_{i j} \geq 1$ for all $i, j$. Then, the map

$$
\phi: V_{01} \oplus V_{02} \oplus V_{12} \rightarrow W_{0} \oplus W_{1} \oplus W_{2}
$$

given as a second order Koszul map (7), is, up to a sign change, equal to the resultant

$$
\mathcal{R}_{\boldsymbol{d}_{0}, \boldsymbol{d}_{1}, \boldsymbol{d}_{2}}\left(f_{0}, f_{1}, f_{2}\right)= \pm \operatorname{det} \phi= \pm \operatorname{det}\left[\begin{array}{ccc}
-\delta_{01}^{0} & -\delta_{02}^{0} & 0 \\
\delta_{01}^{1} & 0 & -\delta_{12}^{1} \\
0 & \delta_{02}^{2} & \delta_{12}^{2}
\end{array}\right]
$$

Proof. From the above discussion and the identification (12), the map in Theorem 3.2 defines the operator (15) (see [42] for more details on these identifications). The corresponding matrix is square, generically non-singular, and equals the determinant of the complex $K_{\bullet}(\boldsymbol{m})$, where $\boldsymbol{m} \in \mathbb{Z}^{2}$ as in (8), therefore it is equal to $\mathcal{R}\left(f_{0}, f_{1}, f_{2}\right)$, up to a sign change.

Example 4.2. We will compute the Koszul resultant matrix for the system of Example 2.1 by adding the following bilinear polynomial

$$
f_{0}=a_{1,1} x y+a_{0,1} y+a_{1,0} x+a_{0,0},
$$

and considering the overdetermined polynomial system $\left\{f_{0}, f_{1}, f_{2}\right\}$. In this case the bidegrees are $\boldsymbol{d}_{0}=(1,1), \boldsymbol{d}_{1}=(1,2)$ and $\boldsymbol{d}_{2}=(2,1)$. The resultant has total degree

$$
\operatorname{deg} \mathcal{R}_{\boldsymbol{d}_{0}, \boldsymbol{d}_{1}, \boldsymbol{d}_{2}}\left(f_{0}, f_{1}, f_{2}\right)=D\left(f_{0}, f_{1}\right)+D\left(f_{0}, f_{2}\right)+D\left(f_{1}, f_{2}\right)=11
$$


and this is also the dimension of the Koszul-type matrix. We compute $\boldsymbol{m}=$ $(3,-1)$ using $(8)$; then (9) becomes

$$
\begin{aligned}
& 0 \rightarrow \mathcal{H}^{1}(1,-4) \oplus \mathcal{H}^{1}(0,-3) \oplus \mathcal{H}^{1}(0,-4) \rightarrow \\
& \mathcal{H}^{1}(2,-2) \oplus \mathcal{H}^{1}(2,-3) \oplus \mathcal{H}^{1}(1,-2) \rightarrow 0 .
\end{aligned}
$$

Using Theorem 4.1 we identify the only map in this complex

$$
\begin{array}{r}
\phi: S_{1}(1) \otimes S_{2}^{*}(2) \oplus S_{1}(0) \otimes S_{2}^{*}(1) \oplus S_{1}(0) \otimes S_{2}^{*}(2) \rightarrow \\
S_{1}(2) \otimes S_{2}^{*}(0) \oplus S_{1}(2) \otimes S_{2}^{*}(1) \oplus S_{1}(1) \otimes S_{2}^{*}(0)
\end{array}
$$

and we obtain the following $3 \times 3$ block matrix containing six primal-dual multiplication blocks

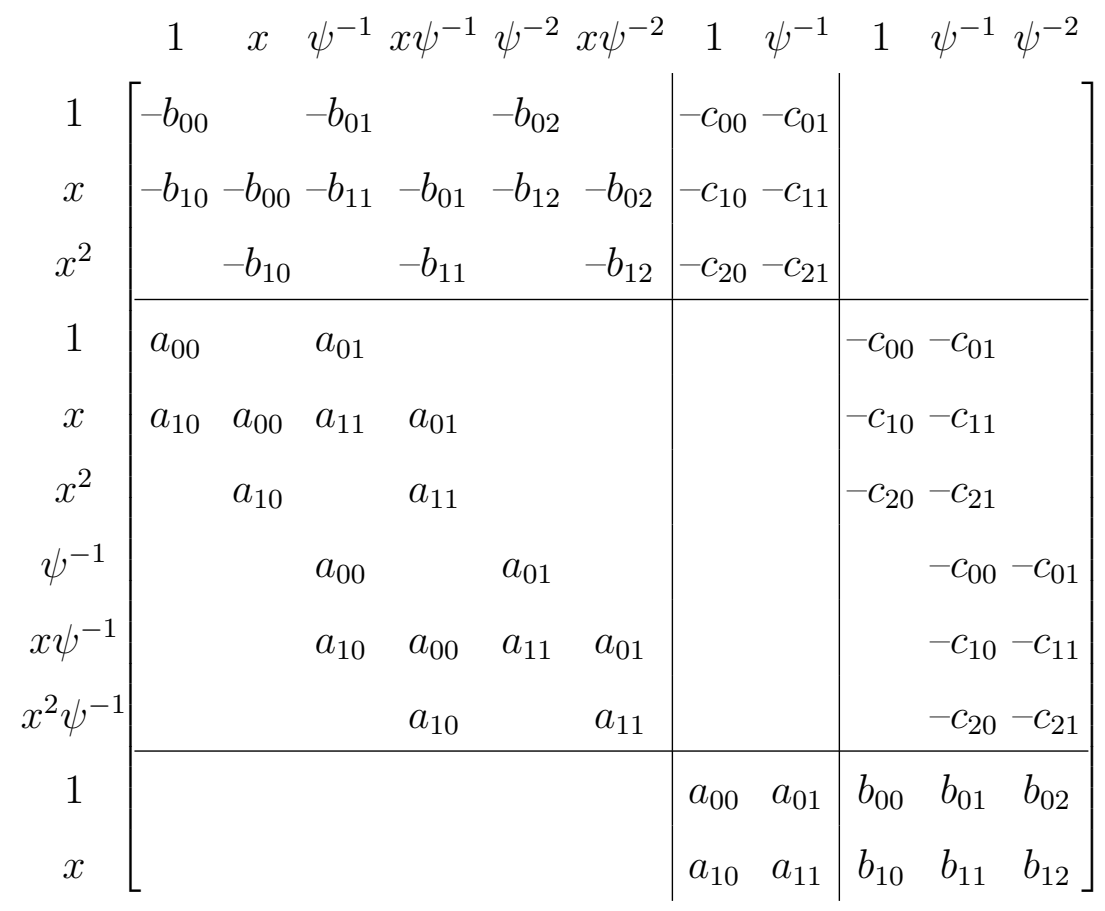

where all the missing entries are equal to zero.

In the general bivariate case the Koszul resultant matrix has a $3 \times 3$ block structure and each block $\delta_{i j}^{r}$ has dimensions

$$
d_{r 2}\left(d_{01}+d_{11}+d_{21}-d_{r 1}\right) \times\left(d_{i 2}+d_{j 2}\right)\left(d_{01}+d_{11}+d_{21}-d_{i 1}-d_{j 1}\right),
$$

for $i, j \in\{0,1,2\}, i<j$ and $r \in\{i, j\}$. 


\subsection{Factorization and multiplicativity}

An interesting consequence of Theorem 4.1 is the following formula that will be useful for the computation of discriminants. Below, the Sylvester resultant of two univariate polynomials $f$ and $g$ in degree $m$ and $n$ (so that $\operatorname{deg}(f) \leq m$ and $\operatorname{deg}(g) \leq n)$ will be denoted by $\operatorname{Res}_{m, n}(f, g)$.

Proposition 4.3. Let $f_{0}, f_{1}, f_{2}$ be bivariate tensor-product polynomials as in (1) such that $d_{i j} \geq 1$ for all $i, j$, then

$$
\begin{aligned}
\mathcal{R}_{\left(d_{01}+1, d_{02}\right), \boldsymbol{d}_{\mathbf{1}}, \boldsymbol{d}_{2}}\left(x f_{0}, f_{1}, f_{2}\right) & = \\
\pm & \operatorname{Res}_{d_{12}, d_{22}}\left(f_{1}(0, y), f_{2}(0, y)\right) \mathcal{R}_{\boldsymbol{d}_{\mathbf{0}}, \boldsymbol{d}_{\mathbf{1}}, \boldsymbol{d}_{\mathbf{2}}}\left(f_{0}, f_{1}, f_{2}\right) .
\end{aligned}
$$

Proof. To prove this formula, we will inspect the matrix of the second order Koszul map $\phi$, as defined in Theorem 4.1, in our particular setting where the first polynomial is here $x f_{0}$, which is of bidegree $\left(d_{01}+1, d_{02}\right)$. More precisely, the matrix of $\phi$ (cf. (15)) takes the form

$$
\left[\begin{array}{ccc}
-\delta_{f_{1}} & -\delta_{f_{2}} & 0 \\
\delta_{x f_{0}} & 0 & -\delta_{f_{2}} \\
0 & \delta_{x f_{0}} & \delta_{f_{1}}
\end{array}\right]
$$

Hereafter, we choose the canonical basis $\left\{1, x, x^{2}, \ldots, x^{a-1}\right\}$ for the space $S_{1}(a)$ in order to analyze the shape of the matrix (17). In particular we will several times decompose $S_{1}(a) \otimes S_{2}^{*}(b)$ as $\left[\mathbb{K} \otimes S_{2}^{*}(b)\right] \oplus\left[S_{1}(a-1) \otimes S_{2}^{*}(b)\right]$ by identifying the vector space generated by $\left\{x, x^{2}, \ldots, x^{a-1}\right\}$ with the vector space generated by $\left\{1, x, x^{2}, \ldots, x^{a-2}\right\}$.

We first consider the nonzero maps in the second row of blocks of (17), which express the maps

$$
V_{01} \stackrel{\delta_{x f_{0}}}{\longrightarrow} W_{1} \quad \text { and } \quad V_{12} \stackrel{-\delta_{f_{2}}}{\longrightarrow} W_{1}
$$

see (13) and (14). Decomposing $W_{1}$ and $V_{12}$ as

$$
W_{1}=\left[\mathbb{K} \otimes S_{2}^{*}\left(d_{12}-1\right)\right] \oplus\left[S_{1}\left(d_{01}+d_{21}-1\right) \otimes S_{2}^{*}\left(d_{12}-1\right)\right]
$$

and

$$
V_{12}=\left[\mathbb{K} \otimes S_{2}^{*}\left(d_{12}+d_{22}-1\right)\right] \oplus\left[S_{1}\left(d_{01}-1\right) \otimes S_{2}^{*}\left(d_{12}+d_{22}-1\right)\right],
$$


the non-zero blocks of the second row of (17) take the form

$$
\left[\begin{array}{c}
0 \\
\hline \delta_{f_{0}}
\end{array}\right] \quad \text { and } \quad\left[\begin{array}{c|c}
M_{-f_{2}(0, y)}^{*} & 0 \\
\hline * & -\delta_{f_{2}}
\end{array}\right] \text {, }
$$

respectively, where the block $M_{-f_{2}(0, y)}^{*}$ denotes the adjoint multiplication map by the polynomial $-f_{2}(0, y)$ from $S_{2}^{*}\left(d_{12}+d_{22}-1\right)$ to $S_{2}^{*}\left(d_{12}-1\right)$, cf. Section 4.1.

In the same spirit, we write

$$
W_{2}=\left[\mathbb{K} \otimes S_{2}^{*}\left(d_{22}-1\right)\right] \oplus\left[S_{1}\left(d_{01}+d_{11}-1\right) \otimes S_{2}^{*}\left(d_{22}-1\right)\right]
$$

and the blocks corresponding to the maps $\delta_{x f_{0}}: V_{02} \rightarrow W_{2}$ and $\delta_{f_{1}}: V_{12} \rightarrow W_{2}$ take the form

$$
\left[\begin{array}{c}
0 \\
\hline \delta_{f_{0}}
\end{array}\right] \text { and }\left[\begin{array}{c|c}
M_{f_{1}(0, y)}^{*} & 0 \\
\hline \star & \delta_{f_{1}}
\end{array}\right],
$$

respectively. Overall, the matrix (17) becomes

$$
\left[\begin{array}{ccc}
-\delta_{f_{1}} & -\delta_{f_{2}} & 0 \\
\delta_{x f_{0}} & 0 & -\delta_{f_{2}} \\
0 & \delta_{x f_{0}} & \delta_{f_{1}}
\end{array}\right]=\left[\begin{array}{c|c|cc|c}
-\delta_{f_{1}} & -\delta_{f_{2}} & 0 & \\
\hline 0 & & M_{-f_{2}(0, y)} & 0 \\
\delta_{f_{0}} & 0 & * & -\delta_{f_{2}} \\
\hline 0 & & M_{f_{1}(0, y)} & 0 \\
\delta_{f_{0}} & 0 & \star & \delta_{f_{1}}
\end{array}\right] \cong\left[\begin{array}{cccc}
M_{-f_{2}(0, y)}^{*} & \multicolumn{2}{|c}{0} \\
M_{f_{1}(0, y)}^{*} & & \\
\hline 0 & -\delta_{f_{1}} & -\delta_{f_{2}} & 0 \\
* & \delta_{f_{0}} & 0 & -\delta_{f_{2}} \\
\star & 0 & \delta_{f_{0}} & \delta_{f_{1}}
\end{array}\right]
$$

where the last congruence is upto a permutation of the rows and the columns of the matrix. The top diagonal block expresses a classical Sylvester map

$$
S_{2}^{*}\left(d_{12}+d_{22}-1\right) \rightarrow S_{2}^{*}\left(d_{12}-1\right) \oplus S_{2}^{*}\left(d_{22}-1\right)
$$

and has determinant equal to $\pm \operatorname{Res}_{d_{12}, d_{22}}\left(f_{1}(0, y), f_{2}(0, y)\right)$. The bottom diagonal block is the Koszul resultant matrix associated to $f_{0}, f_{1}, f_{2}$, and its determinant is equal to $\mathcal{R}_{\boldsymbol{d}_{0}, \boldsymbol{d}_{1}, \boldsymbol{d}_{2}}\left(f_{0}, f_{1}, f_{2}\right)$, by Theorem 4.1. The result follows from the lower triangular form of our global matrix.

The assumption $d_{i j} \geq 1$ in Theorem 4.1 and Proposition 4.3 follows from the main property of the complex (3) presented in Section 3.1. Nevertheless, $\mathcal{R}_{\boldsymbol{d}_{\mathbf{0}}, \boldsymbol{d}_{\mathbf{1}}, \boldsymbol{d}_{\mathbf{2}}}\left(f_{0}, f_{1}, f_{2}\right)$ is well defined without this assumption, that is to say for any non-negative bidegrees (see e.g. [16]). Thus, comparing the formula given in Proposition 4.3 and the multiplicativity property of the resultant (see [16, Corollary 4.6]), which yields the formula

$$
\mathcal{R}_{\left(d_{01}+1, d_{02}\right), \boldsymbol{d}_{\mathbf{1}}, \boldsymbol{d}_{\mathbf{2}}}\left(x f_{0}, f_{1}, f_{2}\right)= \pm \mathcal{R}_{(1,0), \boldsymbol{d}_{\mathbf{1}}, \boldsymbol{d}_{\mathbf{2}}}\left(x, f_{1}, f_{2}\right) \mathcal{R}_{\boldsymbol{d}_{\mathbf{0}}, \boldsymbol{d}_{\mathbf{1}}, \boldsymbol{d}_{\mathbf{2}}}\left(f_{0}, f_{1}, f_{2}\right),
$$


we deduce that $\mathcal{R}_{(1,0), \boldsymbol{d}_{1}, \boldsymbol{d}_{2}}\left(x, f_{1}, f_{2}\right)$ is equal, up to sign, to the Sylvester resultant $\operatorname{Res}_{d_{12}, d_{22}}\left(f_{1}(0, y), f_{2}(0, y)\right)$. More generally, using again several times the multiplicativity property we obtain the following property: For $j=1,2$, set $f_{j}=\sum_{i=0}^{d_{j, 1}} f_{j, i}^{y}(y) x^{i}$. Then, for all $0 \leq k \leq d_{1,1}$ we have that

$$
\begin{aligned}
& \mathcal{R}_{\left(d_{11}, 0\right), \boldsymbol{d}_{1}, \boldsymbol{d}_{2}}\left(x^{k}, f_{1}, f_{2}\right)= \\
& \left.\quad \pm \operatorname{Res}_{d_{12}, d_{22}}\left(f_{1,0}^{y}(y), f_{2,0}^{y}(y)\right)\right)^{k} \operatorname{Res}_{d_{12}, d_{22}}\left(f_{1, d_{11}}^{y}(y), f_{2, d_{21}}^{y}(y)\right)^{d_{11}-k}
\end{aligned}
$$

All the above considerations show that Theorem 4.1 also holds if $d_{02}=0$, and more generally for any non-negative bidegrees $\boldsymbol{d}_{0}, \boldsymbol{d}_{1}, \boldsymbol{d}_{2}$. An interesting consequence is that the formula given in Theorem 4.1 can actually be taken as a defining formula for the resultant $\mathcal{R}_{\boldsymbol{d}_{0}, \boldsymbol{d}_{1}, \boldsymbol{d}_{2}}\left(f_{0}, f_{1}, f_{2}\right)$ without relying on the complex (3), exactly as the Sylvester resultant is usually defined as the determinant of the Sylvester matrix.

\section{Discriminants of TP polynomials}

In this section, we provide new formulæ for computing the discriminant of bivariate tensor-product (TP) polynomials by means of the second order Koszul map given in Theorem 4.1.

\subsection{The discriminant of a single TP polynomial}

We consider a single TP polynomial

$$
f(x, y)=\sum_{i=0}^{d_{1}} \sum_{j=0}^{d_{2}} a_{i, j} x^{i} y^{j}
$$

of bidegree $\boldsymbol{d}=\left(d_{1}, d_{2}\right)$. Assuming $d_{1} \geq 1$ and $d_{2} \geq 1$, we define up to sign the $\boldsymbol{d}$-discriminant of $f$, which we denote by $\Delta_{\boldsymbol{d}}(f)$, as an irreducible polynomial in $\mathbb{Z}[F]$, where $F$ stands for the coefficients of $f$. It has the property that it vanishes after we specialize these coefficients with elements from an algebraically closed field, whenever $f(x, y)$ has a singularity, that is, whenever there is a root of $f$ which satisfies

$$
f=\partial_{x} f=\partial_{y} f=0
$$

(see [25]). This discriminant as a polynomial in the coefficients of $f$, has degree $6 d_{1} d_{2}-4\left(d_{1}+d_{2}-1\right)$. In order to compute it, the principal $\boldsymbol{d}$-determinant, denoted $E(f)$, has been introduced in [25, Chapter 10]. It is defined as the 
resultant

$$
E_{\boldsymbol{d}}(f):=\mathcal{R}_{\boldsymbol{d}, \boldsymbol{d}, \boldsymbol{d}}\left(x \partial_{x} f, y \partial_{y} f, f\right) .
$$

It turns out that this principal $\boldsymbol{d}$-determinant admits a prime factorization in terms of some other discriminants. To describe this formula, we need to introduce the following additional notation. We write $f$ as

$$
f(x, y)=\sum_{i=0}^{d_{1}} \sum_{j=0}^{d_{2}} a_{i, j} x^{i} y^{j}=\sum_{i=0}^{d_{2}} f_{i}^{x}(x) y^{i}=\sum_{j=0}^{d_{1}} f_{j}^{y}(y) x^{j} .
$$

Theorem 5.1 ([25, Theorem 1.2]). With the above notation, we have that

$$
E_{\boldsymbol{d}}(f)= \pm a_{0,0} a_{d_{1}, 0} a_{0, d_{2}} a_{d_{1}, d_{2}} \Delta_{d_{1}}\left(f_{0}^{x}\right) \Delta_{d_{1}}\left(f_{d_{2}}^{x}\right) \Delta_{d_{2}}\left(f_{0}^{y}\right) \Delta_{d_{2}}\left(f_{d_{1}}^{y}\right) \Delta_{\boldsymbol{d}}(f) .
$$

To use the results of the previous section, we introduce the resultant

$$
\mathcal{E}_{d}(f):=\mathcal{R}_{\boldsymbol{d}_{x}, \boldsymbol{d}_{y}, \boldsymbol{d}}\left(\partial_{x} f, \partial_{y} f, f\right)
$$

where $\boldsymbol{d}_{x}:=\left(d_{1}-1, d_{2}\right)$ and $\boldsymbol{d}_{y}:=\left(d_{1}, d_{2}-1\right)$. We recall that we can compute this resultant as the determinant of the second order Koszul resultant matrix $\phi$ given in Theorem 4.1 .

Lemma 5.2. With the above notation, we have that

$$
\mathcal{E}_{\boldsymbol{d}}(f)=\mathcal{R}_{\boldsymbol{d}_{x}, \boldsymbol{d}_{y}, \boldsymbol{d}}\left(\partial_{x} f, \partial_{y} f, f\right)= \pm a_{d_{1}, d_{2}} \Delta_{d_{1}}\left(f_{d_{2}}^{x}\right) \Delta_{d_{2}}\left(f_{d_{1}}^{y}\right) \Delta_{\boldsymbol{d}}(f) .
$$

Proof. Using Proposition 4.3, we obtain that

$$
E_{\boldsymbol{d}}(f)= \pm \operatorname{Res}_{d_{2}, d_{2}}\left(y \partial f_{0}^{y}, f_{0}^{y}\right) \mathcal{R}_{\boldsymbol{d}_{x}, \boldsymbol{d}, \boldsymbol{d}}\left(\partial_{x} f, y \partial_{y} f, f\right)
$$

and using the classical property of the Sylvester resultant we get that

$$
\operatorname{Res}_{d_{2}, d_{2}}\left(y \partial f_{0}^{y}, f_{0}^{y}\right)=\operatorname{Res}_{1, d_{2}}\left(y, f_{0}^{y}\right) \operatorname{Res}_{d_{2}-1, d_{2}}\left(\partial f_{0}^{y}, f_{0}^{y}\right)= \pm a_{0,0} a_{0, d_{2}} \Delta_{d_{2}}\left(f_{0}^{y}\right) .
$$

Therefore, we deduce that

$$
E_{\boldsymbol{d}}(f)= \pm a_{0,0} a_{0, d_{2}} \Delta_{d_{2}}\left(f_{0}^{y}\right) \mathcal{R}_{\boldsymbol{d}_{x}, \boldsymbol{d}, \boldsymbol{d}}\left(\partial_{x} f, y \partial_{y} f, f\right) .
$$

Now, using again Proposition 4.3, we get that

$$
\mathcal{R}_{\boldsymbol{d}_{x}, \boldsymbol{d}, \boldsymbol{d}}\left(\partial_{x} f, y \partial_{y} f, f\right)= \pm \operatorname{Res}_{d_{1}-1, d_{1}}\left(\partial_{x} f_{0}^{x}, f_{0}^{x}\right) \mathcal{E}_{\boldsymbol{d}}(f)= \pm a_{d_{1}, 0} \Delta_{d_{1}}\left(f_{0}^{x}\right)
$$

so that we obtain the equality

$$
E_{\boldsymbol{d}}(f)= \pm a_{0,0} a_{d_{1}, 0} a_{0, d_{2}} \Delta_{d_{1}}\left(f_{0}^{x}\right) \Delta_{d_{2}}\left(f_{0}^{y}\right) \mathcal{E}_{\boldsymbol{d}}(f) .
$$

The claimed formula then follows by comparison with Theorem 5.1. 
The interest in $\mathcal{E}_{\boldsymbol{d}}(f)$ stems from the fact that it possesses a determinantal representation, which is much more simple to describe and manipulate, compared to $\Delta_{\boldsymbol{d}}(f)$ itself. Indeed, we are in position to compute $\mathcal{E}(f)$ using the Koszul resultant matrix, by plugging in the coefficients of $f$ and its partial derivatives. We emphasize that the univariate discriminants in the formula of the previous lemma can be computed using the Sylvester resultants, namely

$\operatorname{Res}_{d_{1}-1, d_{1}}\left(\partial_{x} f_{d_{2}}^{x}, f_{d_{2}}^{x}\right)=a_{d_{1}, d_{2}} \Delta_{d_{1}}\left(f_{d_{2}}^{x}\right), \quad \operatorname{Res}_{d_{2}-1, d_{2}}\left(\partial_{y} f_{d_{1}}^{y}, f_{d_{1}}^{y}\right)=a_{d_{1}, d_{2}} \Delta_{d_{2}}\left(f_{d_{1}}^{y}\right)$,

so that, informally, we have the following determinantal formula

$$
\Delta_{\boldsymbol{d}}(f)=\frac{a_{d_{1}, d_{2}} \mathcal{E}_{\boldsymbol{d}}(f)}{\operatorname{Res}_{d_{1}-1, d_{1}}\left(\partial_{x} f_{d_{2}}^{x}, f_{d_{2}}^{x}\right) \operatorname{Res}_{d_{2}-1, d_{2}}\left(\partial_{y} f_{d_{1}}^{y}, f_{d_{1}}^{y}\right)}
$$

where each term in the right hand side of the above equality can be computed as a single matrix determinant.

Formula (20) can be actually improved if one allows derivation by the homogenizing variables of $x$ and $y$ over $\mathbb{P}^{1} \times \mathbb{P}^{1}$. More precisely, denote by $\bar{x}$, respectively $\bar{y}$, the homogenizing variable of $x$, respectively $y$, over $\mathbb{P}^{1}$. Let $F$ be the homogenization of the polynomial $f$, that is

$$
\begin{aligned}
F(\bar{x}, x ; \bar{y}, y) & =\sum_{i=0}^{d_{1}} \sum_{j=0}^{d_{2}} a_{i, j} \bar{x}^{d_{1}-i} x^{i} \bar{y}^{d_{2}-j} y^{j} \\
& =\sum_{i=0}^{d_{2}} F_{i}^{x}(\bar{x}, x) \bar{y}^{d_{2}-i} y^{i}=\sum_{j=0}^{d_{1}} F_{j}^{y}(\bar{y}, y) \bar{x}^{d_{1}-j} x^{j}
\end{aligned}
$$

From now on, we will adopt indifferently the homogeneous or the inhomogeneous notation in resultants and discriminants, as for instance $\Delta_{\boldsymbol{d}}(f)$ or $\Delta_{\boldsymbol{d}}(F)$ to denote the discriminant of $f$; moreover we define

$$
\mathcal{E}_{\boldsymbol{d}}^{x}(f):=\mathcal{R}_{\boldsymbol{d}_{x}, \boldsymbol{d}_{x}, \boldsymbol{d}_{y}}\left(\partial_{\bar{x}} F, \partial_{x} F, \partial_{y} F\right) .
$$

Proposition 5.3. With the above notation we have that

$$
\mathcal{E}_{\boldsymbol{d}}^{x}(f)=\mathcal{R}_{\boldsymbol{d}_{x}, \boldsymbol{d}_{x}, \boldsymbol{d}_{y}}\left(\partial_{\bar{x}} F, \partial_{x} F, \partial_{y} F\right)= \pm d_{1}^{d_{1} d_{2}+\left(d_{1}-2\right)\left(d_{2}-1\right)} \Delta_{d_{1}}\left(f_{d_{2}}^{x}\right) \Delta_{\boldsymbol{d}}(f) .
$$

Proof. First by homogeneity property of the resultant with respect to the coefficients of each of its input polynomial, we get that

$$
\mathcal{R}_{\boldsymbol{d}_{x}, \boldsymbol{d}_{y}, \boldsymbol{d}}\left(\partial_{x} F, \partial_{y} F, d_{1} F\right)=d_{1}^{d_{1} d_{2}+\left(d_{1}-1\right)\left(d_{2}-1\right)} \mathcal{E}_{\boldsymbol{d}}(f) .
$$

On the other hand, by Euler's formula $d_{1} F=x \partial_{x} F+\bar{x} \partial_{\bar{x}} F$ so that

$$
\mathcal{R}_{\boldsymbol{d}_{x}, \boldsymbol{d}_{y}, \boldsymbol{d}}\left(\partial_{x} F, \partial_{y} F, d_{1} F\right)=\mathcal{R}_{\boldsymbol{d}_{x}, \boldsymbol{d}_{y}, \boldsymbol{d}}\left(\partial_{x} F, \partial_{y} F, \bar{x} \partial_{\bar{x}} F\right)
$$


Now, using Proposition 4.3 we deduce that

$$
\begin{aligned}
& \mathcal{R}_{\boldsymbol{d}_{x}, \boldsymbol{d}_{y}, \boldsymbol{d}}\left(\partial_{x} F, \partial_{y} F, \bar{x} \partial_{\bar{x}} F\right)= \\
& \quad \pm \operatorname{Res}_{d_{1}, d_{1}-1}\left(\partial_{x} F(0,1 ; \bar{y}, y), \partial_{y} F(0,1 ; \bar{y}, y)\right) \mathcal{R}_{\boldsymbol{d}_{x}, \boldsymbol{d}_{y}, \boldsymbol{d}_{x}}\left(\partial_{x} F, \partial_{y} F, \partial_{\bar{x}} F\right) .
\end{aligned}
$$

But from the definition, $\partial_{x} F(0,1 ; \bar{y}, y)=d_{1} F_{d_{1}}^{y}$ and $\partial_{y} F(0,1 ; \bar{y}, y)=\partial_{y} F_{d_{1}}^{y}$ so that

$$
\operatorname{Res}_{d_{1}, d_{1}-1}\left(\partial_{x} F(0,1 ; \bar{y}, y), \partial_{y} F(0,1 ; \bar{y}, y)\right)= \pm d_{1}^{d_{2}-1} a_{d_{1}, d_{2}} \Delta_{d_{2}}\left(F_{d_{1}}^{y}\right) .
$$

Gathering the above computations, we deduce that

$$
d_{1}^{d_{1} d_{2}+\left(d_{1}-2\right)\left(d_{2}-1\right)} \mathcal{E}_{\boldsymbol{d}}(F)= \pm a_{d_{1}, d_{2}} \Delta_{d_{2}}\left(F_{d_{1}}^{y}\right) R_{\boldsymbol{d}_{x}, \boldsymbol{d}_{y}, \boldsymbol{d}_{x}}\left(\partial_{x} F, \partial_{y} F, \partial_{\bar{x}} F\right)
$$

and then the claimed formula follows by combining this equality together with Lemma 5.2.

The formula we just proved can be seen as an extension of the Demazure's formula $^{2}$ for the discriminant of a homogeneous polynomial (see [13, Proposition 11] and [25, Chapter 13, Proposition 1.7]): let $h\left(x_{0}, x_{1}, x_{2}\right)$ be a homogeneous polynomial of degree $d \geq 2$ in the variables $x_{0}, x_{1}, x_{2}$, then

$$
d^{d^{2}-3(d-1)} \Delta_{d}(h)= \pm \operatorname{Res}_{d-1, d-1, d-1}\left(\partial_{x_{0}} h, \partial_{x_{1}} h, \partial_{x_{2}} h\right)
$$

where the resultant ${ }^{3}$ is considered in $\mathbb{P}^{2}$ and $\Delta_{d}(h)$ denotes the discriminant of $h$ as a homogeneous polynomial of degree $d \geq 2$.

We emphasize that here again we obtain a determinantal formula for the discriminant of $f$ where each term can be computed as a single determinant, namely

$$
d_{1}^{d_{1} d_{2}+\left(d_{1}-2\right)\left(d_{2}-1\right)} \Delta_{\boldsymbol{d}}(f)= \pm \frac{a_{d_{1}, d_{2}} \mathcal{E}_{\boldsymbol{d}}^{x}(f)}{\operatorname{Res}_{d_{1}, d_{1}-1}\left(f_{d_{2}}^{x}, \partial_{x} f_{d_{2}}^{x}\right)} .
$$

We also mention that a similar formula obviously holds if one replaces the use of the partial derivative $\partial_{\bar{x}} F$ by $\partial_{\bar{y}} F$ in Proposition 5.3.

For practical computations, we would like to have the smallest matrix size, which is equivalent to the smallest degree of the extra factor in the determinant. Therefore, whenever $d_{1}<d_{2}$ the use of $\mathcal{E}_{\boldsymbol{d}}^{x}(f)$ is preferable for computing $\Delta_{\boldsymbol{d}}(f)$, whereas in the opposite case $\mathcal{E}_{\boldsymbol{d}}^{y}(f)$ should be chosen instead.

$\overline{2}$ As far as we know, this formula goes back to an unpublished Bourbaki manuscript by Demazure dated of 1969 .

3 Let us mention that determinantal formulæ do arise from Weyman's complex for this resultant. However, all formulæ have (parts with) order strictly greater than two. 


\subsection{The discriminant of two TP polynomials}

We can generalize this approach to the computation of mixed discriminants [19, $24]$. The mixed discriminant, denoted $\Delta_{\boldsymbol{d}_{1}, \boldsymbol{d}_{2}}\left(f_{1}, f_{2}\right)$, of a bivariate tensorproduct system of equations $f_{1}=f_{2}=0$ is defined (under genericity assumptions) as the unique (up to sign) integer, irreducible polynomial in the coefficients of $f_{1}, f_{2}$ which vanishes whenever the system has a non-degenerate multiple root.

We consider the Jacobian polynomial

$$
J(x, y)=\partial_{x} f_{1} \partial_{y} f_{2}-\partial_{y} f_{1} \partial_{x} f_{2},
$$

and we denote its bidegree as $\boldsymbol{q}=\left(q_{1}, q_{2}\right)=\left(d_{11}+d_{21}-1, d_{12}+d_{22}-1\right)$.

Proposition 5.4. With the above notation, we have that

$$
\begin{aligned}
& \mathcal{R}_{\boldsymbol{d}_{1}, \boldsymbol{d}_{2}, \boldsymbol{q}}\left(f_{1}, f_{2}, J\right)= \\
& \quad \pm \operatorname{Res}_{d_{11}, d_{21}}\left(f_{1, d_{12}}^{x}, f_{2, d_{22}}^{x}\right) \operatorname{Res}_{d_{12}, d_{22}}\left(f_{1, d_{11}}^{y}, f_{2, d_{21}}^{y}\right) \Delta_{\boldsymbol{d}_{1}, \boldsymbol{d}_{2}}\left(f_{1}, f_{2}\right)
\end{aligned}
$$

where we use a similar notation as in (19).

Proof. We adopt the homogeneous notation for the polynomials, $\bar{x}$ denoting the homogenizing variable of $x$ and $\bar{y}$ the homogenizing variable of $y$. The homogenization of $f_{1}$ and $f_{2}$ are denoted $F_{1}$ and $F_{2}$ respectively.

Now, in addition to the Jacobian determinant

$$
J(\bar{x}, x ; \bar{y}, y)=\partial_{x} F_{1} \partial_{y} F_{2}-\partial_{y} F_{1} \partial_{x} F_{2},
$$

we also introduce the other Jacobian determinant

$$
\bar{J}(\bar{x}, x ; \bar{y}, y)=\partial_{\bar{x}} F_{1} \partial_{y} F_{2}-\partial_{y} F_{1} \partial_{\bar{x}} F_{2}
$$

Using the Euler formula with respect to the set of homogeneous variables $\bar{x}, x$, we get that

$$
\bar{x} \bar{J}-x J \in\left(d_{11} f_{1}, d_{21} f_{2}\right) .
$$

It follows that

$$
\mathcal{R}_{\boldsymbol{d}_{1}, \boldsymbol{d}_{2},\left(q_{1}+1, q_{2}\right)}\left(f_{1}, f_{2}, x J\right)=\mathcal{R}_{\boldsymbol{d}_{1}, \boldsymbol{d}_{2},\left(q_{1}+1, q_{2}\right)}\left(f_{1}, f_{2}, \bar{x} \bar{J}\right) .
$$

Using the multiplicativity property and the irreducibility of generic resultants we deduce that

$$
\mathcal{R}_{\boldsymbol{d}_{1}, \boldsymbol{d}_{2},(1,0)}\left(f_{1}, f_{2}, \bar{x}\right)= \pm \operatorname{Res}_{d_{12}, d_{22}}\left(f_{1, d_{11}}^{y}, f_{2, d_{21}}^{y}\right)
$$


divides $\mathcal{R}_{\boldsymbol{d}_{1}, \boldsymbol{d}_{2}, \boldsymbol{q}}\left(f_{1}, f_{2}, J\right)$. By using a similar argument with the couple of homogeneous variables $\bar{y}, y$ we also get that

$$
\mathcal{R}_{\boldsymbol{d}_{1}, \boldsymbol{d}_{2},(0,1)}\left(f_{1}, f_{2}, \bar{y}\right)= \pm \operatorname{Res}_{d_{11}, d_{21}}\left(f_{1, d_{12}}^{x}, f_{2, d_{22}}^{x}\right)
$$

divides $\mathcal{R}_{\boldsymbol{d}_{1}, \boldsymbol{d}_{2}, \boldsymbol{q}}\left(f_{1}, f_{2}, J\right)$, so that the product (of generically coprime polynomials)

$$
\operatorname{Res}_{d_{11}, d_{21}}\left(f_{1, d_{12}}^{x}, f_{2, d_{22}}^{x}\right) \operatorname{Res}_{d_{12}, d_{22}}\left(f_{1, d_{11}}^{y}, f_{2, d_{21}}^{y}\right)
$$

divides $\mathcal{R}_{\boldsymbol{d}_{1}, \boldsymbol{d}_{2}, \boldsymbol{q}}\left(f_{1}, f_{2}, J\right)$.

In order to conclude the proof, we compute the partial degrees of these resultants since we know that $\Delta_{\boldsymbol{d}_{1}, \boldsymbol{d}_{2}}\left(f_{1}, f_{2}\right)$ also divides $\mathcal{R}_{\boldsymbol{d}_{1}, \boldsymbol{d}_{2}, \boldsymbol{q}}\left(f_{1}, f_{2}, J\right)$. The bidegree of the two factors above with respect to $\left(f_{1}, f_{2}\right)$ is $\left(d_{21}, d_{11}\right)$ and $\left(d_{22}, d_{12}\right)$, respectively. Moreover, $\Delta_{\boldsymbol{d}_{1}, \boldsymbol{d}_{2}}\left(f_{1}, f_{2}\right)$ has degree $2\left(d_{11} d_{22}+d_{12} d_{21}+\right.$ $\left.d_{j 1} d_{j 2}-d_{j 1}-d_{j 2}\right)$ with respect to $f_{i}$, for any $\{i, j\}=\{1,2\}$, cf. [24]. Finally we can directly derive the bidegree of the resultant, which (in terms of Bézout numbers $(2))$ is $D\left(f_{1}, f_{2}\right)+D\left(J, f_{j}\right)$ with respect to $f_{i}$, where $\{i, j\}=\{1,2\}$. We have

$$
D\left(f_{1}, f_{2}\right)+D\left(J, f_{j}\right)=2\left(d_{11} d_{22}+d_{12} d_{21}+d_{j 1} d_{j 2}\right)-d_{j 1}-d_{j 2},
$$

where $\{i, j\}=\{1,2\}$. By degree reasons the claimed formula must hold up to multiplication by a nonzero multiplicative constant. To show that this constant is equal to \pm 1 , it is enough to exhibit a particular example where this is the case. For instance, taking $f_{1}$, resp. $f_{2}$, as the product of $d_{11}$, resp. $d_{21}$, generic linear forms in $\bar{x}$ and $x$ and $d_{12}$, resp. $d_{22}$, generic linear forms in $\bar{y}$ and $y$, the resultant $\mathcal{R}_{\boldsymbol{d}_{1}, \boldsymbol{d}_{2}, \boldsymbol{q}}\left(f_{1}, f_{2}, J\right)$ is a primitive polynomial, which can easily be seen by applying the multiplicativity property of the resultant.

\subsection{Some illustrative examples}

Next, we compute discriminants of some low-degree cases, to demonstrate the use of our formulæ. All the computations in this section were prepared using the Maple package Polyonimo ${ }^{4}$.

- Bilinear case $\left(d_{1}, d_{2}\right)=(1,1)$. We consider a bilinear polynomial, say $f$, and its partial derivatives $\partial_{x} f=a_{1,0}+a_{1,1} y, \partial_{y} f=a_{0,1}+a_{1,1} x$. It is well known that the discriminant of $f$ equals the determinant of the associated coefficient matrix of the bilinear form $f$, that is $\Delta_{(1,1)}(f)=a_{0,0} a_{1,1}-a_{0,1} a_{1,0}$. We can

$\overline{4 \text { https }}$ //github.com/filiatra/polyonimo 
compute the $(2 \times 1$ block $)$ matrix

$$
\mathcal{E}_{\boldsymbol{d}}^{x}(f)=\mathcal{E}_{\boldsymbol{d}}^{y}(f)=\operatorname{det}\left[\begin{array}{cc}
-a_{1,1} & -a_{1,0} \\
a_{0,1} & a_{0,0}
\end{array}\right]=-\Delta_{(1,1)}(f),
$$

which is the matrix of the map

$$
\mathcal{H}^{1}(0,-3) \rightarrow \mathcal{H}^{1}(0,-2)^{\oplus 2}
$$

For a comparison, we also compute

$$
\mathcal{E}_{\boldsymbol{d}}(f)=\operatorname{det}\left[\begin{array}{rrr}
-a_{1,1} & -a_{0,1} & 0 \\
a_{1,0} & a_{0,0} & a_{1,0} \\
a_{1,1} & a_{0,1} & a_{1,1}
\end{array}\right]=-a_{1,1} \Delta_{(1,1)}(f)
$$

corresponding to the map

$$
\mathcal{H}^{1}(-2,0) \oplus \mathcal{H}^{1}(-3,0) \rightarrow \mathcal{H}^{1}(-2,1) \oplus \mathcal{H}^{1}(-2,0) .
$$

Here, the extra factor $a_{1,1}$ appears, as Lemma 5.2 predicts.

- Linear-quadratic case: $\left(d_{1}, d_{2}\right)=(1,2)$. We have

$$
\begin{aligned}
& \mathcal{E}_{\boldsymbol{d}}(f)=a_{1,2}\left(a_{1,1}^{2}-4 a_{1,2} a_{1,0}\right) \Delta_{(1,2)}(f) \\
& \mathcal{E}_{\boldsymbol{d}}^{y}(f)=-2^{2}\left(a_{1,1}{ }^{2}-4 a_{1,2} a_{1,0}\right) \Delta_{(1,2)}(f) \\
& \mathcal{E}_{\boldsymbol{d}}^{x}(f)=\Delta_{(1,2)}(f)
\end{aligned}
$$

and the final discriminant polynomial is

$$
\begin{gathered}
\Delta_{(1,2)}(f)=a_{0,2}^{2} a_{1,0}^{2}+a_{0,0}^{2} a_{1,2}^{2}+a_{0,0} a_{1,1}^{2} a_{0,2}+a_{1,2} a_{0,1}^{2} a_{1,0} \\
-2 a_{1,0} a_{1,2} a_{0,2} a_{0,0}-a_{0,2} a_{1,0} a_{1,1} a_{0,1}-a_{0,1} a_{0,0} a_{1,1} a_{1,2} .
\end{gathered}
$$

- Biquadratic case $\left(d_{1}, d_{2}\right)=(2,2)$. Using Sylvester's matrix we compute $\Delta_{2}\left(a_{0,2}+a_{1,2} y+a_{2,2} y^{2}\right)$ and $\Delta_{2}\left(a_{2,0}+a_{2,1} x+a_{2,2} x^{2}\right)$. The final reduced principal determinants are

$$
\begin{aligned}
& \mathcal{E}_{\boldsymbol{d}}^{x}(f)=-2^{4}\left(a_{1,2}^{2}-4 a_{2,2} a_{0,2}\right) \Delta_{(2,2)}(f), \\
& \mathcal{E}_{\boldsymbol{d}}^{y}(f)=-2^{4}\left(a_{2,1}^{2}-4 a_{2,2} a_{2,0}\right) \Delta_{(2,2)}(f),
\end{aligned}
$$

and the polynomial $\Delta_{(2,2)}(f)$ has 1010 terms.

- Consider a polynomial of bidegree $\left(d_{1}, d_{2}\right)=(2,3)$. The most compact formula that we can compute is

$$
\mathcal{E}_{\boldsymbol{d}}^{x}(f)=-2^{8}\left(a_{1,3}^{2}-4 a_{2,3} a_{0,3}\right) \Delta_{(2,3)}(f)
$$


where $\Delta_{(2,3)}(f)$ has 230372 terms.

-Regarding the mixed discriminant, as a first example we consider

$$
\begin{aligned}
f_{1} & =a_{0,0}+a_{1,0} x+a_{0,1} y+a_{1,1} x y \\
f_{2} & =b_{0,0}+b_{1,0} x+b_{0,1} y+b_{1,1} x y \\
J & =a_{1,0} b_{0,1}-b_{1,0} a_{0,1}+ \\
& +\left(a_{1,0} b_{1,1}-b_{1,0} a_{1,1}\right) x+\left(a_{1,1} b_{0,1}-b_{1,1} a_{0,1}\right) y .
\end{aligned}
$$

Note that the Jacobian is missing the monomial $x y$. Nevertheless our Koszul matrix is generically non-singular. We obtain its (factorized) determinant as

$\mathcal{R}_{(1,1),(1,1),(1,1)}\left(f_{1}, f_{2}, J\right)=-\left(a_{1,0} b_{1,1}-b_{1,0} a_{1,1}\right)\left(a_{1,1} b_{0,1}-b_{1,1} a_{0,1}\right) \Delta_{(1,1),(1,1)}\left(f_{1}, f_{2}\right)$

where

$$
\begin{aligned}
\Delta_{(1,1),(1,1)}\left(f_{1}, f_{2}\right) & =b_{1,0}^{2} a_{0,1}^{2}+4 a_{0,1} a_{1,0} b_{0,0} b_{1,1}+4 a_{0,0} a_{1,1} b_{0,1} b_{1,0} \\
& -2 a_{0,1} a_{0,0} b_{1,0} b_{1,1}-2 a_{0,1} b_{0,0} b_{1,0} a_{1,1}+b_{0,1}^{2} a_{1,0}^{2} \\
& -2 a_{1,0} a_{0,0} b_{0,1} b_{1,1}-2 a_{1,0} b_{0,0} b_{0,1} a_{1,1}+a_{0,0}^{2} b_{1,1}^{2} \\
& -2 b_{0,0} a_{0,0} a_{1,1} b_{1,1}-2 b_{0,1} b_{1,0} a_{1,0} a_{0,1}+b_{0,0}^{2} a_{1,1}^{2} .
\end{aligned}
$$

In [6, Example 2.3] this discriminant is computed as the hyperdeterminant of the Cayley matrix. Indeed, in their Theorem 2.1 the authors associate the mixed discriminant with the Cayley matrix, depicted in [25, Ch. 14, Prop. 1.7].

-A second example we are going to treat is $\boldsymbol{d}_{1}=(1,2)$ and $\boldsymbol{d}_{2}=(2,1)$. In this case we have the two extra factors in $\mathcal{R}_{\boldsymbol{d}_{1}, \boldsymbol{d}_{2},(2,2)}\left(f_{1}, f_{2}, J\right)$ :

$$
\begin{aligned}
& \operatorname{Res}_{2,1}\left(f_{1,1}^{y}, f_{2,2}^{y}\right)=a_{1,2} b_{2,0}{ }^{2}-b_{2,1} a_{1,1} b_{2,0}+b_{2,1}{ }^{2} a_{1,0} \\
& \operatorname{Res}_{1,2}\left(f_{1,2}^{x}, f_{2,1}^{x}\right)=b_{0,1} a_{1,2}{ }^{2}-a_{0,2} b_{1,1} a_{1,2}+b_{2,1} a_{0,2}{ }^{2}
\end{aligned}
$$

and the main factor $\Delta_{(1,2),(2,1)}$ has 16333 terms.

In practice, we would not expand the matrix determinant to obtain the discriminant polynomial. A rank test on the involved matrices suffices to decide whether the system is singular.

\section{Acknowledgments}

ET is partially supported by an FP7 Marie Curie Career Integration Grant and ANR JCJC GALOP. 


\section{References}

[1] R. Bott. Homogeneous vector bundles. Annals of Mathematics, 66(2): 203-248, 1957.

[2] L. Busé. Resultants of determinantal varieties. Journal of Pure and Applied Algebra, 193(13):71 - 97, 2004.

[3] L. Busé, M. Elkadi, and B. Mourrain. Using projection operators in computer aided geometric design. Comtemporary Mathematics, 334:321$342,2003$.

[4] J. F. Canny, E. Kaltofen, and L. Yagati. Solving systems of nonlinear polynomial equations faster. In Proc. ISSAC, pages 121-128, 1989.

[5] E. Cattani and A. Dickenstein. Solving Polynomial Equations: Foundations, Algorithms, and Applications, chapter Introduction to residues and resultants, pages 1-61. Springer Berlin Heidelberg, Berlin, Heidelberg, 2005. ISBN 978-3-540-27357-8.

[6] E. Cattani, M. A. Cueto, A. Dickenstein, S. Di Rocco, and B. Sturmfels. Mixed discriminants. Mathematische Zeitschrift, 274(3):761-778, 2013.

[7] A. Chtcherba and D. Kapur. Resultants for unmixed bivariate polynomial systems produced using the Dixon formulation. Journal of Symbolic Computation, 38(2):915 - 958, 2004. ISSN 0747-7171. doi: http://dx. doi.org/10.1016/j.jsc.2003.12.001.

[8] A. D. Chtcherba and D. Kapur. Conditions for exact resultants using the dixon formulation. In Proc. Int'l Symposium on Symbolic and Algebraic Computation, ISSAC, pages 62-70, 2000.

[9] D. Cox and E. Materov. Tate resolutions for Segre embeddings. Algebra $\& 5$ Number Theory, 2(5):523-550, 2008.

[10] C. D'Andrea. Macaulay style formulas for sparse resultants. Transactions of the American Mathematical Society, 354(7):2595-2629, 2002.

[11] C. D'Andrea and A. Dickenstein. Explicit formulas for the multivariate resultant. J. Pure and Applied Algebra, 164(1-2):59-86, 2001.

[12] C. D'Andrea and I. Z. Emiris. Hybrid sparse resultant matrices for bivariate systems. In Proc. of the 2001 Int'l Symposium on Symbolic and Algebraic Computation, ISSAC, pages 24-31, 2001.

[13] M. Demazure. Résultant, discriminant. LEnseignement Mathématique, 58(3):333-373, 2012.

[14] A. Dickenstein and I. Z. Emiris. Multihomogeneous resultant formulae by means of complexes. Journal of Symbolic Computation, 36(3):317-342, 2003.

[15] A. Dickenstein, I. Z. Emiris, and A. Karasoulou. SAGA - Advances in ShApes, Geometry, and Algebra: Results from the Marie Curie Initial Training Network, chapter Plane Mixed Discriminants and Toric Jacobians, pages 105-121. Springer Int'l Publishing, Cham, 2014. ISBN 978-3-319-08635-4.

[16] C. DAndrea and M. Sombra. A poisson formula for the sparse resultant. Proceedings of the London Mathematical Society, 110(4):932-964, 2015. 
[17] M. Elkadi and A. Galligo. Systems of three polynomials with two separated variables. In Proc. Int'l Symposium on Symbolic and Algebraic Computation, ISSAC, pages 159-166, 2007.

[18] I. Emiris and R. Vidunas. Root counts of semi-mixed systems, and an application to counting Nash equilibria. In Proc. ACM ISSAC, pages 154-161, Kobe, Japan, 2014. ACM Press.

[19] I. Z. Emiris and A. Karasoulou. Future Vision and Trends on Shapes, Geometry and Algebra, chapter Sparse Discriminants and Applications, pages 55-71. Springer London, London, 2014. ISBN 978-1-4471-6461-6.

[20] I. Z. Emiris and A. Mantzaflaris. Multihomogeneous resultant formulae for systems with scaled support. Journal of Symbolic Computation, 47 (7):820-842, 2012.

[21] I. Z. Emiris and B. Mourrain. Matrices in elimination theory. Journal of Symbolic Computation, 28(1):3-44, 1999.

[22] I. Z. Emiris and V. Y. Pan. Symbolic and Numeric Methods for Exploiting Structure in Constructing Resultant Matrices. Journal of Symbolic Computation, 33(4):393-413, Apr. 2002.

[23] I. Z. Emiris and V. Y. Pan. Improved algorithms for computing determinants and resultants. J. of Complexity, 21(1):43-71, Feb. 2005.

[24] A. Esterov. The discriminant of a system of equations. Advances in Mathematics, 245:534 - 572, 2013.

[25] I. Gelfand, M. Kapranov, and A. Zelevinsky. Discriminants, Resultants and Multidimensional Determinants. Birkhäuser, Boston, 1994.

[26] R. Hartshorne. Algebraic Geometry. Springer, New York, 1977.

[27] A. Khetan. The resultant of an unmixed bivariate system. Journal of Symbolic Computation, 36(3-4):425-442, 2003.

[28] A. Khetan, N. Song, and R. Goldman. Sylvester A-resultants for Bivariate Polynomials with Planar Newton Polygons. In Proc. Int'l Symposium on Symbolic and Algebraic Computation, ISSAC, pages 205-212, 2004.

[29] F. S. Macaulay. Some formulæin elimination. Proceedings of the London Mathematical Society, s1-35(1):3-27, 1902.

[30] A. Mantzaflaris and B. Mourrain. Deflation and certified isolation of singular zeros of polynomial systems. In Proc. ACM ISSAC, pages 249256, 2011.

[31] A. Mantzaflaris and B. Mourrain. Singular zeros of polynomial systems. In T. Dokken and G. Muntingh, editors, Advances in Shapes, Geometry, and Algebra, volume 10 of Geometry and Computing, pages 77-103. Springer, 2014.

[32] A. Mantzaflaris and E. Tsigaridas. Resultants and discriminants for bivariate tensor-product polynomials. In In Proc. Int'l Symposium on Symbolic and Algebraic Computation (ISSAC), page 8, 2017.

[33] N. McCoy. On the resultant of a system of forms homogeneous in each of several sets of variables. Trans. AMS, 35(1):215-233, 1933.

[34] A. McLennan. The maximum number of real roots of a multihomogeneous system of polynomial equations. Contributions to Algebra and Geometry, 
40(2):343-350, 1999.

[35] E. Miller and B. Sturmfels. Combinatorial commutative algebra, volume 227. Springer Science \& Business Media, 2004.

[36] T. Muir. The resultant of a set of lineo-linear equations. Proc. Royal Soc. of South Africa, 2(1):373-380, 1910.

[37] H. Schenck, D. Cox, and A. Dickenstein. A case study in bigraded commutative algebra. Syzygies \& Hilbert Functions, 254:67-112, 2007.

[38] B. Sturmfels and A. Zelevinsky. Multigraded resultants of Sylvester type. J. Algebra, 163(1):115-127, 1994.

[39] J. Sylvester. On the degree and weight of the resultant of a multipartite system of equations. Proc. Royal Soc. of London, 12:674-676, 1862.

[40] J. Weyman. Calculating discriminants by higher direct images. Trans. of AMS, 343(1):367-389, 1994.

[41] J. Weyman. Cohomology of Vector Bundles and Syzygies. Cambridge Univ. Press, 2003. [Cambridge Tracts in Mathematics 149].

[42] J. Weyman and A. Zelevinsky. Determinantal formulas for multigraded resultants. J. Alg. Geom., 3(4):569-597, 1994.

[43] M. Zhang and R. Goldman. Rectangular corner cutting and sylvester A-resultants. In Proc. Int'l Symposium on Symbolic and Algebraic Computation, ISSAC, pages 301-308, 2000. 